\title{
BRIDGING THE COMMUNICATION GAP BETWEEN BUSINESS AND IT WITH BEHAVIOUR-DRIVEN DEVELOPMENT: A CUSTOM APPROACH
}

\section{VANDENBROECK DAVID ${ }^{1}$, KEMME MIEKE ${ }^{2}$, STEEGMANS ELKE $^{3}$, FRANSEN WANNES ${ }^{4}$, LAUWEREYS NICOLAS ${ }^{5} \&$ THIELEMANS FREDERIK ${ }^{6}$}

\author{
Department of Applied Informatics, University College Leuven Limburg (UCLL), Geldenaaksebaan 335, 3001 \\ Leuven, Vlaams-Brabant, Belgium.
}

\begin{abstract}
Behaviour-Driven Development (BDD) was first coined by Dan North in an attempt to bridge the communication gap that tends to exists between business and IT. Where Test-Driven Development (TDD) is aimed at building the software right, BDD aims at building the right software. While TDD aims to uncover coding mistakes early in the production stages, BDD rather aims to uncover and avoid misunderstandings, even before any line of code has been written. As misunderstandings are generally caused by a lack of communication, BDD enforces several practices to enforce communicating and discussing all aspects of a functionality by expressing and discussing its behaviour by means of examples. In order to make the discussions accessible to the business, the behaviour is exemplified in a ubiquitous and domain-specific language. Doing so, the business is able to be more involved, making it easier to spot misunderstandings, even before development has started. However, leveraging the benefits of BDD is not as obvious as it might seem. BDD is not always adopted properly and the three consecutive phases of BDD (i.e., discovery, formulation and automation) are not always executed correctly, or not at all. In this paper, we propose a custom approach to each of the three phases in BDD, based on three maturity levels, in order to lower the barrier for adopting the practices of BDD and maximizing their full potential.

KEYWORDS: BDD, Behaviour-Driven Development, TDD, Test-Driven Development, Ubiquitous Language, Communication, Domain-Specific Language
\end{abstract}

Received: Jun 08, 2020; Accepted: Jun 28, 2020; Published: Aug 31, 2020; Paper Id.: IJMPERDJUN2020987

\section{INTRODUCTION}

Behaviour-Driven Development (BDD) came to life when Dan North (2006) noticed the need for clarifications for tests in projects where Test-Driven Development (TDD) was adopted. Dan North proposed to write test methods in a more comprehensible manner, creating a mind switch from test to behaviour and making the intent of the tests more clear to testers and developers. In addition, a template for translating acceptance criteria into scenarios was conceptualized utilizing a Given-When-Then (GWT) structure. Thanks to these scenarios, the gap between acceptance criteria and tests became abridged[1]. BDD is therefore largely seen as an addition to TDD[2]. Where the practices of TDD especially aim at reducing the amount of bugs on unit and integration level, the practices of BDD aim to, in addition, reduce the amount of bugs at the acceptance level, focussing on the requirements itself[3]. BDD, however, is not always adopted properly and the benefits of BDD might get outweighed by the effort made. One of the main causes for BDD not being adopted properly, is due to the misconception that BDD is a tool, rather than a method. In addition, BDD has a few pitfalls that organisations have to deal with when they initially adopt 
BDD, which adds an additional barrier to adopt BDD successfully[4]. In order to try lowering the barrier for adopting BDD, we have conceptualized a custom BDD matrix, allowing for a gradual approach and the highest return on investment based on the level of maturity for each of the three consecutive phases of BDD.

\section{Three Consecutive Phases of BDD}

BDD consists of three consecutive phases that can be adopted, which are called the "discovery", "formulation" and "automation" phase respectively. Each phase provides input for the next, meaning that when a certain phase is not executed correctly, incorrect input is used for the successive phases. Skipping a phase would work counterproductive, as each phase is dependent on input that should have been gathered in the skipped phase. Skipping a phase would therefor result in mixing two phases together, causing for both phases to be executed inadequately. In the first phase of discovery, the most important work needs to be done, namely discovering the unknown, rectifying misunderstandings and creating a common language. It is therefore highly recommended to start with the discovery phase, followed by formulation and automation respectively. In addition, the discovery phase highlights the core of BDD, i.e., collaboration. While the discovery phase is the phase that requires the least technical skills, as it needs to allow for everyone to be able to contribute, it does require a lot of effort from everyone on the team in order to be executed adequately. When the essence of the discovery phase is not understood correctly, it will again result in incorrect input for the successive phases. The discovery phase encompasses defining a user story, gathering requirements and specifying those requirements with acceptance criteria. In order to avoid misunderstandings, the acceptance criteria are exemplified until all acceptance criteria are clear to everyone. In BDD, this process is ideally executed by at least the three amigos, commonly during an example mapping session. In the following phase, formulation, the examples that have been framed during the discovery phase, in order to exemplify the acceptance criteria, are translated (formulated) into scenarios using a Given-When-Then structure. The scenarios are then grouped in.feature-files based on the functionality they exemplify. In the third phase, automation, the formulated scenarios are transformed into readable steps that are used for test automation. Automated tests based on the formulated scenarios now also result in living documentation that is automatically generated, which can be seen as one of the most useful benefits of BDD.

\section{BEHAVIOUR-DRIVEN DEVELOPMENT IN PRACTICE}

\section{Misconceptions on Behaviour-Driven Development}

While there is extensive documentation on the automation phase of BDD to be found, documentation or studies on the two preceding phases is less prevalent, keeping the core characteristics of BDD rather hidden[5]. The prevalence of tool support for BDD and their focus on test automation might also provide a certain bias toward the core intent of BDD.Hence, there seems to be a common misconception that BDD is a tool rather than a method.Souza et al., even mention that BDD does not have a clear definition and that there is no unanimous understanding[6]. Due to perceiving BDD as a tool, the focus on writing scenarios with the intention of collaboration, communication and deriving a common understanding, is surpassed by formulating scenarios with the sole intention of automating them and using them, for example,in the context of an end-to-end testing solution. While this type of usage could be beneficial in certain situations, it is not the core intent of BDD. However, even in academic literature, BDD is often also referred to when it is mostly just about test automation, as was done in the paper by Ali[7], for example.In addition, BDD might also be perceived as a 100\% test coverage solution, which is actually near to impossible to reach [8]. 
In theory, BDD seems to be the holy grail, while in practice, BDD proves to be a lot more difficult to adopt. One of the main causes on this account is that BDD actually alters the traditional approach to software development[9]. BDD introduces high involvement of every role in the team, including the product owner, to the process, which poses another challenge [4]. Adopting a new software development method on its own already requires a lot of effort[10], which is not any different for BDD. All team members should be able, and willing, to collaborate in order to make BDD worthwhile.

In certain cases, for example, testers and developers are not used to working closely together, but rather reside in separate teams, like the V-Shaped model[11] proposes. When developers and testers do work closely together, there might still be conflicts that are in the way of proper collaboration[12]. In order to adopt BDD properly, the entire team must therefore not only master the new practices that come with BDD, but in most cases also drastically alter the way they collaborate with each other. Therefore, a development team should require everyone's support and the time and resources in order to accomplish the altered approach to software development. The biggest advantage of BDD is that all those who are involved are able to collaboratively contribute[4]. Not willing to collaborate evades the goal of BDD, making it difficult to successfully adopt its practices.

\section{Pitfalls of Behaviour-Driven Development}

Even in case there is optimal collaboration within the development team, BDD still requires aninitial learning curve[9], which organisations need to overcome to benefit from the advantages of BDD [4]. In order to avoid adopting BDD improperly, the team should deep-dive into BDD before they decide to suddenly adopt BDD in its entirety for one of their projects. If $\mathrm{BDD}$ is not properly adopted from the beginning, chances are it will alienate certain team members from following the practices of BDD, making it difficult to uphold and maintain BDD in the future[9]. Important is that the team is aware of the misconceptions of BDD and that BDD is not being adopted for the wrong reason (e.g., end-to-end testing). Mastering all three phases of BDD, discovery, formulation and automation, requires extensive practice, as each phase has its own challenges, which is not always feasible due to a possible lack of time or resources.

\section{A Proposal for A Custom Approach Based on Maturity}

Given the misconceptions and pitfalls that surround BDD, it proves difficult to adopt BDD correctly in its entirety. In order to facilitate the adoption of BDD, we therefore propose a custom approach to adopting BDD, based on a certain level of maturity. For each of the three phases in BDD, three maturity levels have been contrived. For each level of maturity, a minimum amount of effort is required in order to complete that specific phase and to be able to advance to the next, as presented below in fig. 1. 
Level 1

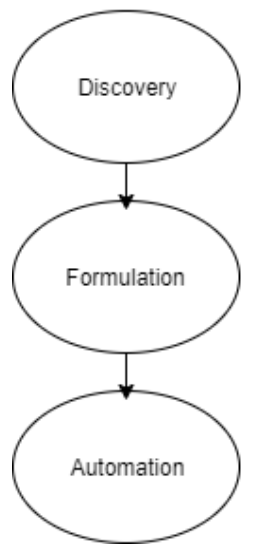

Level 2
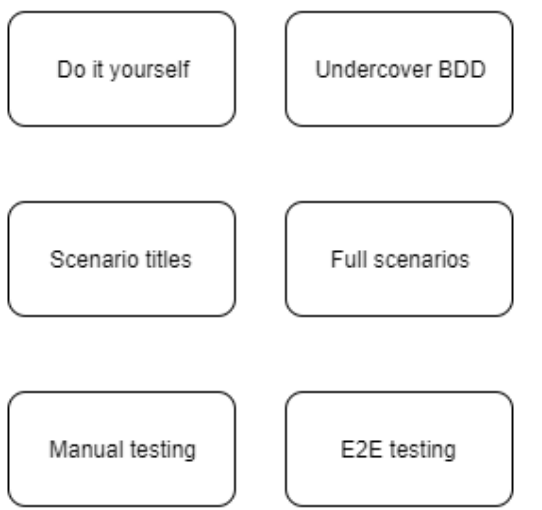

Level 3

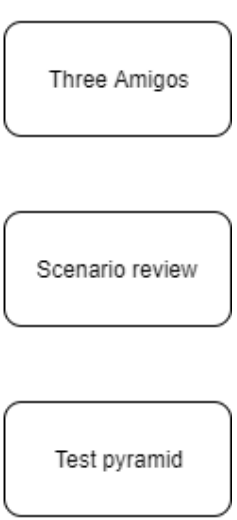

Figure 1: Custom BDD matrix.

BDD starts with the most important phase, i.e., discovery. In this phase it is important to deliberately try and discover what is still unknown, while creating a shared understanding by specifying acceptance criteria, or business rules, by examples. Ideally, at the highest level of maturity, it is suggested that at least the "three amigos" (i.e., Tester, Developer and Business Analyst) explore a user story together in a requirements workshop (e.g., in an example mapping session). By doing so, the three amigos can each bring their own experience to the table, broadening the exploration area of the user story and shortening feedback loops that would otherwise be required. However, as mentioned before, the simultaneous collaboration is not always possible. Therefore, on a lower level, it might be easier at first to only involve those that were initially interested in adopting BDD and to adopt BDD in an "undercover" manner. Instead of organising specific three amigos meetings for a requirements workshop, try to bring the requirements workshop to regular meetings in which at least the three amigos are present, without mentioning the goal of the meeting. If it is not possible to bring at least the three amigos together for a requirements workshop, or if the "undercover BDD" is not working due to the three amigos not actively looking for examples to specify the acceptance criteria, on the lowest level, you could still perform a requirements workshop on your own, where you specify the acceptance criteria with examples on your own. After doing so, you can try and discuss the examples with the three amigos. The lowest level is, however,far from ideal, as the examples are based on only one person's point of view.

The second phase of BDD encompasses formulating the examples that have been gathered during a requirements workshop into business readable Gherkin (GWT) scenarios. The goal of this phase is to verify that the tester and developer both understand how the behaviour of the application should function. In an ideal situation, the scenarios are written in pair by the developer and tester and are then reviewed by at least the product owner. At this level of maturity, it is required that the product owner is interested in reading the formulated scenarios, in order for the scenarios to be reviewed thoroughly. This step thus requires a good understanding of how scenarios should be written, in order not to alienate the product owner from reviewing the scenarios. While formulating scenarios might show to seem very simple, it is a rather tedious task.Oliveira et al., even dedicated a research paper to propose a question-based checklist to assess the quality of a scenario[13]. At a lower level, you might therefore practice writing good scenarios first, ideally with the entire team. If there is not much interest in the scenarios yet by the team, it might still be beneficial to start with providing only the scenarios that are of interest to the team (level 2), or to even only share the titles of the examples that have been specified in the discovery phase (level 1). Even only the scenario titles could already serve as a guideline for automating tests, especially when already practicing TDD. 
In the third phase, automation, the input of the formulation phase is used for automating tests. Ideally the reviewed scenarios are integrated in the full test stack, covering mainly Unit and integration tests, in addition to programmer tests. It's also possible that certain tests will not be automated, but are at least marked as being tested manually. By integrating the scenarios in the full test stack, there is an automatic system that warns if certain behaviour does not work as intended (anymore), providing for the benefit of living documentation. When integration in the full test stack is not possible yet, on a second level, it might be a good idea to start using the scenarios only for end-to-end testing. However, when using the scenarios solely for end-to-end testing, don't forget to keep in mind that theintent of formulating scenarios will always be enabling collaboration, not end-to-end testing. Executing end-to-end tests just doesn't always require extensive technical skills and can avoid the need to alter the development environment. Instead, the tests can be automated in a separate environment, which also provides for a system that warns if tests are out of date, so that there still is the benefit of living documentation. On a lower level, if there are also no resources to automate the scenarios as end-toend tests, it would still be of use to utilize the scenarios to execute manual tests. Giving the formulated scenarios a purpose ensures that the scenarios are decently written and that the practices of BDD will be properly upheld. As a minimal effort in the lowest level of maturity, the scenarios are all still best used to execute manual tests and kept as documentation in order to maximize its usefulness.

\section{DISCUSSIONS}

Conceptualizing a custom approach to adopting BDD might lower the barrier for companies to adopt BDD as a whole, as it allows for a structured approach based on the team's current needs and their situation[10]. In addition, it should allow the team to more easily grow into the practices of BDD without requiring extensive knowledge on BDD upfront. While undergoing the different maturity levels, the team is able to upskill their BDD knowledge hands-on, with sufficient margin for practice. However, it is still a first attempt to a conceptualization of different approaches based on certain maturity levels. Further research and practical experience with the custom BDD matrix might still refine the matrix based on retrieving the most benefits out of each level/phase with the lowest effort or skill required.

\section{CONCLUSIONS}

The custom BDD matrix should provide more room for organisations wanting to adopt BDD and should avoid their teams to become alienated to the practices of BDD. Meanwhile, the custom BDD matrix highlights the importance of each phase in BDD, as well as the pitfalls that teams have to overcome when adopting BDD practices. It also highlights that BDD is rather a set of practices that can be adopted separately, instead of all at once. However, further empirical research should reveal the practicality of applying the custom BDD matrix to organisations wanting to adopt BDD in their projects. In addition, we would recommend adopting BDD in only a limited amount of sprints first, for a limited set of user stories. Doing so, the team will be less dependent on their knowledge of BDD and are provided with even more room for practice. This will also provide an initial idea of the practices of BDD and its benefits. If the practices of BDD don't provide for any added value to your project, it might be of interest to try a different development method.

\section{ACKNOWLEDGEMENTS}

The authors gratefully acknowledge everyone who participated in the preliminary interviews, the project leads who agreed to adopt BDD intro their projects, the students who have adopted BDD for their project and, lastly, the Digital Solutions centre of expertise of the University College Leuven-Limburg (UCLL), for making our research on BDD possible. 


\section{REFERENCES}

1. North D: Introducing BDD. Dan North \& Associates 2006.

2. Moe M: Comparative Study of Test-Driven Development TDD, Behavior-Driven Development BDD and Acceptance Test-Driven Development ATDD. International Journal of Trend in Scientific Research and Development 2019; Vol.3, $231-234$.

3. de Carvalho RA, de Carvalho e Silva FL, Manhães RS, de Oliveira GL: Implementing Behavior Driven Development in an Open Source ERP. Enterprise Information Systems of the Future2013; 242-249.

4. Pereira L, Sharp H, de Souza C, Oliveira G, Marczak S, Bastos R: Behavior-driven development benefits and challenges: reports from an industrial study. Proceedings of the 19th International Conference on Agile Software Development: Companion 2018; 1-4.

5. Solis C, Wang X: A Study of the Characteristics of Behaviour Driven Development. 2011 37th EUROMICRO Conference on Software Engineering and Advanced Applications 2011; 383-387.

6. Souza P, Prado A, Lopes de Souza W, Pereira S, Pires L: Combining Behaviour-Driven Development with Scrum for Software Development in the Education Domain 2017; 449-458.

7. Ali Z: Behavior-Driven Development as an Error-Reduction Practice for Mobile Application Testing, 2019.

8. Diepenbeck M, Soeken M, Grobe D, Drechsler R: Towards automatic scenario generation from coverage information. 2013 8th International Workshop on Automation of Software Test (AST) 2013; 82-88.

9. Binamungu LP, Embury SM, Konstantinou N: Maintaining behaviour driven development specifications: Challenges and opportunities. 2018 IEEE 25th International Conference on Software Analysis, Evolution and Reengineering (SANER)2018; 175-184.

10. Nikitina N, Kajko-Mattsson M: Guiding the adoption of software development methods. ACM International Conference Proceeding Series, 2014.

11. Ruparelia N: Software development lifecycle models. ACM SIGSOFT Software Engineering Notes 2010;35:8-13.

12. Zhang X, Stafford T, Dhaliwal J, Gillenson M, Moeller G: Sources of Conflict between Developers and Testers in Software Development. Information \& Management 2014; 51:13-26.

13. Oliveira G, Marczak S, Moralles C: How to Evaluate BDD Scenarios' Quality? Proceedings of the XXXIII Brazilian Symposium on Software Engineering 2019; 481-490. 